\title{
Industrial Membrane Processes for the Removal of VOCs from Water and Wastewater
}

\author{
Hisham A. Maddah
}

\begin{abstract}
This paper illustrates the role of different membrane technologies in the treatment of VOCs from water and wastewaters. Emerging membrane technologies such as pervaporation (PV) and membrane distillation (MD) gained scientists' attention as a promising way for water treatment. We have studied and discussed today's advanced membranes: PV membranes which included polysiloxaneimide (PSI) and polydimethylsiloxane (PDMS), and MD membranes which included electro-spun nanofiber membrane and vacuum membrane distillation (VMD). Studied membranes showed great potential for the treatment of VOCs from water. However, it was found that certain factors like feed temperature, downstream pressure, component volatility (Henry's constant) and VOCs concentration must be considered in the design phase to achieve the ideal treatment.
\end{abstract}

Index Terms - Membrane, Distillation, Pervaporation, Organics, Water Treatment, VOCs.

\section{INTRODUCTION}

Available fresh water sources on earth are limited to rivers, lakes, snowpack, wetlands, and aquifers [1]. The demand for drinking water is increasing rapidly since the world's population is estimated to increase by another $40-50 \%$ by 2050 [2]. Polluted water and industrial wastewater pose potential risks to humans and the environment [3]. Membrane systems are considered as excellent technologies for wastewater treatment since they are capable to remove organic pollutants which can harm the environment. However, captured organics may reduce water diffusion and flux rates of water across the membrane; accumulation of organic materials on the membrane surface results in membrane biofouling which decreases membrane production efficiency $[2,4,5]$.

Currently, many industrial applications involve the use of organic solvents in their technological processes in order to have products such as refrigerants, plastics, adhesives, paints, and petroleum products [6]. Huge amounts of wastewaters that are contaminated with volatile organic compounds (VOCs) are produced on a daily basis from several industries; causing serious environmental impacts which lead to numerous chronic health diseases [7,8]. Peng et al. (2003) reported that man-made VOCs could also come from other sources such as municipal waste, traffic and agricultural operations. VOCs from industrial effluents and municipal wastes may leak into groundwater and contaminate a major possible drinking water source [11]. Therefore, increased attention from governments has been devoted to treating VOCs from industrial and municipal wastewaters. VOCs of particular interest include petroleum-based solvents, such as

Hisham A. Maddah, Department of Chemical Engineering, King Abdulaziz University, Rabigh, 25732, Saudi Arabia benzene, toluene, ethyl benzene, and xylenes (BTEX) and chlorinated solvents, such as trichloroethylene (TCE) and tetrachloroethylene (PCE) [11].

Treatment or recovery of VOCs compounds from wastewaters is usually accomplished by distillation processes. However, Peng et al. (2003) have mentioned that the water solubilities of VOCs are usually low [11]. In other words, the ability of VOCs to be dissolved in water is very small and therefore there will be very low amounts of dissolved VOCs in water; making the conventional distillation process not economically feasible for the removal of VOCs from water [11]. On top of that, the distillation process requires high energy for separation which will increase the total treatment cost [6].

There are other possible treatment methods for removing VOCs which include air stripping, adsorption by activated carbon, advanced oxidation, anaerobic/aerobic biological treatment, and membrane technology [11]. Most of the conventional treatment processes such as air stripping and adsorption have many shortcomings and limitations for the removal of VOCs. For example, air stripping is susceptible to fouling and turns the problem into an air pollution issue. Air stripping is not effective for low volatile compounds (low Henry's constants). On the other hand, activated carbon adsorption needs costly regeneration steps making it very expensive and may not be suitable for high levels of VOCs. Advanced oxidation is only efficient for specific compounds, very expensive and may form harmful byproducts while biological treatment is time-consuming and effective only at low VOCs levels $[6,11]$.

\section{MEMBRANE PROCESSES FOR VOCS REMOVAL FROM WATER}

Recent advancements in membrane processes captured the attention of governments to utilize membrane technologies in the removal of pollutants such as VOCs from water and wastewaters. Researchers Eustache and Histi (1981); Rautenbach and Albrecht (1985) defined the membrane technology as a separation process that rejects contaminants by exclusion or diffusion due to the differences in size, shape, electrical charge, concentration, partial pressure or solubility between membrane materials and components in water $[9,10]$. Significant progress in membrane technology and its industrial applications has been observed since the 1970s. However, Kujawa et al. (2015) realized that pervaporation (PV) and membrane distillation (MD) were not studied carefully for water treatment applications until the early 1980s; where much of the attention previously was devoted to reverse osmosis (RO) and ultrafiltration (UF) [6].

\section{A. Membrane pervaporation}

Separation of mixtures of liquids by partial vaporization 
through a non-porous or porous membrane is known as pervaporation. Pervaporation process allows organics to be selectively separated from aqueous systems by placing the liquid stream, containing organics, in contact with one side of a nonporous selective membrane while a vacuum is applied to the other side. The selected organics in the liquid stream passes through three consecutive steps: sorption of organics into the membrane, permeation through the membrane and evaporation into the vapor phase where the vapor is then condensed for further disposal [11].

The driving force of separation is associated with the difference in the partial pressures of the components on the two sides and not the volatility difference of the components in the feed. In other words, transport through the membrane is induced by maintaining a lower vapor pressure on the permeate side (gas) than the feed side (liquid). The membrane upstream side is usually kept at ambient pressure whereas the membrane downstream side is put under vacuum to initiate the transport process. This process is used by a number of industries for several and different applications, including purification and analysis, due to its simplicity and in-line nature [11]. Fig. 1 illustrates the PV treatment mechanism.

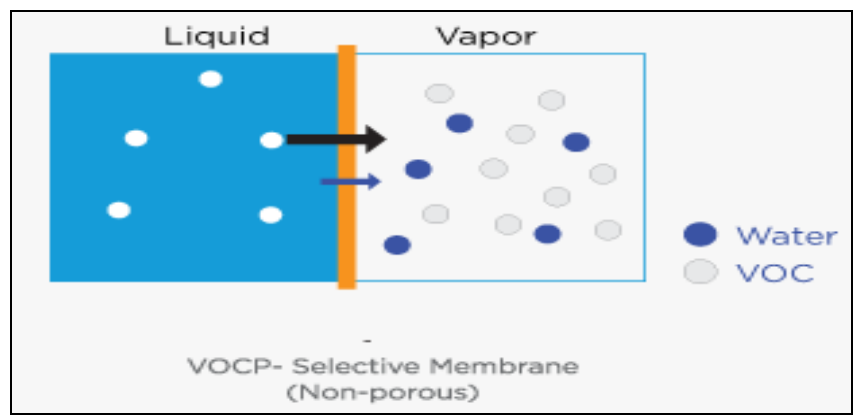

Fig. 1. Pervaporation mechanism in the treatment of VOCs from water [12].

In 1996, ZENON Technologies Incorporation proposed a full-scale demonstration of their ZENON cross-flow pervaporation system for groundwater treatment as shown in Fig. 2. The pervaporation unit was mainly designed to remove trichloroethene (TCE) from groundwater with influent water flow rates of 2 to $11 \mathrm{gpm}$ and a maximum allowed TCE concentration of $250 \mathrm{mg} / \mathrm{L}$. ZENON system was capable of removing up to $97.3 \%$ of TCE from groundwater [13].

ZENON cross-flow pervaporation system has been already applied on a large scale for various industrial applications including the removal of VOCs from wastewater and groundwater. The pervaporation process followed the same discussed technique for separating contaminates in which partial pressures of different components were manipulated. In ZENON cross-flow pervaporation system, contaminated water was typically pumped from an equalization tank through a prefilter that removed debris. Then, contaminated water entered a heat exchanger which raised the water temperature to approximately $75^{\circ} \mathrm{C}$. The pervaporation module containing the membranes received the heated contaminated water where organics were adsorbed and attached into the membranes. Meanwhile, a vacuum was applied at the membrane downstream side of the system which allowed organics to diffuse through the membrane as vapor. The retentate (liquid) that did not move through the membrane was discharged from the system and would be free of VOCs. However, the permeate (vapor) would pass through a condenser to concentrate VOCs contaminants and separate the liquid into aqueous and organic phases. The aqueous phase was recycled for treatment while the organic phase was discharged [13].

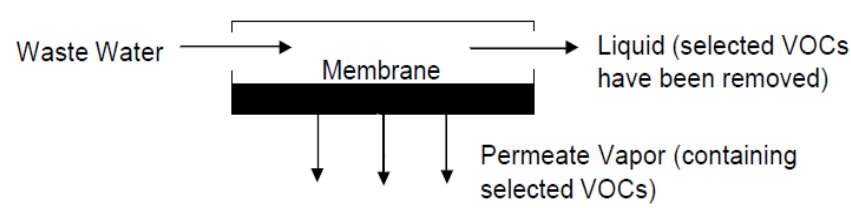

Fig. 2. A full-scale demonstration of ZENON cross-flow pervaporation system for VOCs treatment [13].

\section{B. Membrane distillation}

Couffin et al. (1998) defined the MD process as a thermally-driven separation, in which only vapor molecules transfer through a microporous hydrophobic membrane. In the MD process, water is transferred through a hydrophobic membrane by a difference in vapor pressure which is induced by the temperature difference. In other words, the driving force of the process corresponds to both partial pressure and thermal gradients. The difference in vapor pressure allows water in gas form to pass the membrane pores while water molecules in liquid form are rejected by the hydrophobic membranes. The liquid/vapor equilibrium across the two sides of the membrane controls the process selectivity [14] Fig. 3 below shows the discussed principle of the MD process.

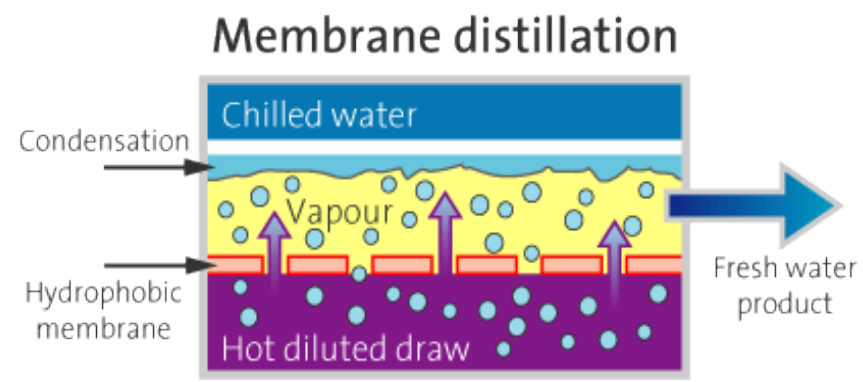

Fig. 3. Principle of MD separation process [15].

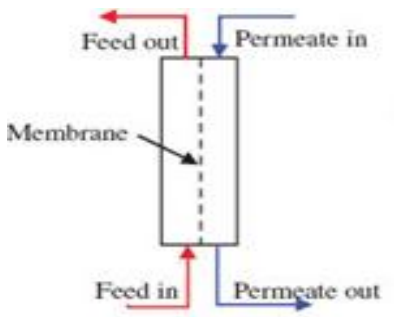

DCMD

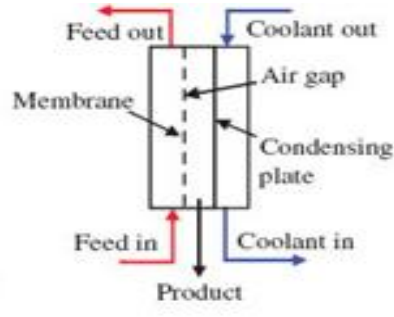

AGMD

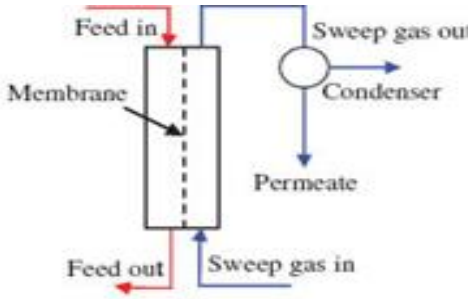

SGMD

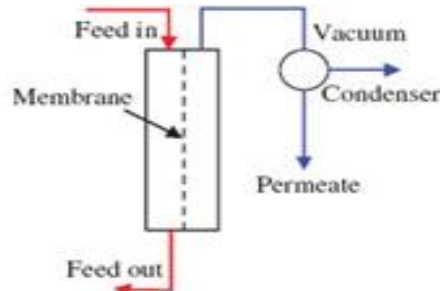

VMD

Fig. 4. Different membrane distillation configurations [16]. 
There are four common membrane distillation configurations where the separation is triggered by various distillate or permeate triggers such as aqueous solution, air gap, vacuum or sweep gas [16]. Fig. 4 shows the most common MD configurations which are direct contact membrane distillation (DCMD), air gap membrane distillation (AGMD), sweep gas membrane distillation (SGMD) and vacuum membrane distillation (VMD).

DCMD configuration involves a microporous membrane that separates the membrane feed side (water) from the permeate side (distillate). In DCMD configuration, the permeate side or the cooling liquid is put in direct contact with the membrane surface. Both sides of the membrane have a liquid-vapor interface, but only vapors can pass to the permeate side. Passing vapors will condense at the permeate side since there is a higher temperature on the feed water side compared to the permeate side. However, AGMD configuration introduces an air gap between the membrane and the permeate side to separate the cooling chamber from the membrane. In other words, only the feed solution is put in contact with the membrane in AGMD configuration. In AGMD, condensation of passing vapors across the membrane occurs on a cooler surface than DCMD because of the presence of the air gap which also reduces the energy required for condensation [16].

The configuration SGMD is often called as air stripping MD since passing vapors (permeate) are removed by a flow of sweeping gas in which condensation is done externally in a separate unit. The blown air will move parallel to the membrane surface in order to remove volatile organics from water vapors. VMD has a different technique for the removal of VOCs in the passing vapors where the permeate side is kept at vacuum by a vacuum pump and vapors are subsequently condensed externally. The VMD method is effective for the removal of volatile compounds from water and it has been applied recently for the treatment of RO brines at seawater desalination plants [16].

\section{ADVANCED MEMBRANE TYPES FOR VOCS REMOVAL FROM WATER}

\section{A. Pervaporation membranes}

Polysiloxaneimide (PSI) membrane: Three polysiloxaneimide membranes were prepared and compared by Chang et al. (2000) to check their performances toward the removal of organic compounds or VOCs. The degree of membrane sorption for a pure organic compound was calculated from Eq. (1). Total membrane permeation flux and pervaporation selectivity (separation factor) have been determined by using Eq. (2) and Eq. (3), respectively. Experimental results showed high sorption for more hydrophobic organics and high selectivity/flux towards hydrophobic organic compounds for the three studied membranes. Membrane selectivity, Eq. (3), is defined as the ratio of permeability of species $i$ with relation to species $j$. Selectivity coefficient determines the ability of the membrane to separate species $i$ from $j$; a selectivity of one indicates the membrane has no potential to separate the two gases since both gasses will diffuse equally [17].

$$
\begin{gathered}
\Phi_{p}(w t . \%)=\frac{W_{w e t}-W_{d r y}}{W_{d r y}} \times 100 \\
l\left(\mathrm{~kg} / \mathrm{m}^{2} \cdot \mathrm{hr}\right)=Q /(A \times T) \\
a_{0 / \mathrm{f}}^{p}=\frac{Y_{0} / Y_{W}}{X_{0} / X_{W}}
\end{gathered}
$$

The effect of various parameters such as feed concentration, feed temperature, permeate pressure, and membrane thickness on pervaporation performance of the PSI membranes was studied for $0.05 \mathrm{wt}$. $\%$ of methylene dichloride (MC) in a water mixture at $30^{\circ} \mathrm{C}$, Table 1 . It was proved that increasing applied pressure would decrease permeation flux, but enhance membrane selectivity. The same effect was observed with increasing membrane thickness and/or feed organic concentration. Nevertheless, increasing feed temperature reduced membrane diffusion resistance towards MC and increased the entropy of mixing. Accordingly, the permeation flux increased, but pervaporation selectivity decreased [17]. Fig. 5 determines the effect of the concentration of MC on permeation flux and pervaporation selectivity of the PSI membranes for $0.05 \mathrm{wt}$. $\%$ of $\mathrm{MC} /$ water mixture at $30^{\circ} \mathrm{C}$.

Table 1. Effect of various parameters on pervaporation performance of the PSI membranes for $0.05 \mathrm{wt}$. \% of $\mathrm{MC} /$ water mixture at $30^{\circ} \mathrm{C}$.

\begin{tabular}{|c|c|c|c|}
\hline Parameter & Action & Permeation Flux & Selectivity \\
\hline Pressure & $\begin{array}{c}\text { Increasing permeate pressure } \\
\text { from } 1.35 \text { to } 15 \text { Torr }\end{array}$ & $\begin{array}{c}\text { Decreases slightly } \\
\downarrow\end{array}$ & $\begin{array}{c}\text { Increases linearly } \\
\uparrow\end{array}$ \\
\hline Temperature & Increasing feed temperature & $\begin{array}{c}\text { Increases } \\
\uparrow\end{array}$ & $\begin{array}{c}\text { Decreases } \\
\downarrow\end{array}$ \\
\hline Thickness & $\begin{array}{c}\text { Increasing membrane } \\
\text { thickness }\end{array}$ & $\begin{array}{c}\text { Decreases } \\
\downarrow\end{array}$ & $\begin{array}{c}\text { Increases slightly } \\
\uparrow\end{array}$ \\
\hline Concentration & Increasing feed concentration & $\begin{array}{c}\text { Decreases } \\
\downarrow\end{array}$ & $\begin{array}{c}\text { Increases } \\
\uparrow\end{array}$ \\
\hline
\end{tabular}

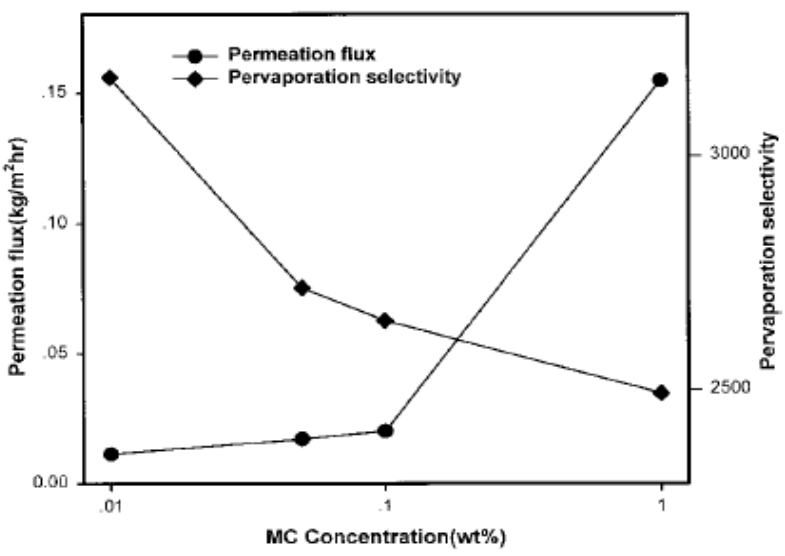

Fig. 5. Effect of the concentration of the organic compound (MC) on permeation flux and pervaporation selectivity of PSI membranes at $30^{\circ} \mathrm{C}$ [17]

Polydimethylsiloxane (PDMS) membrane: Graft polymers are segmented copolymers with a linear backbone of one composite and randomly distributed branches of 
another composite. Shinoda and Matyjaszewski (2001) and Hawker et al. (1997) determined that alkyl grafting could be achieved by either homogenous or heterogeneous distribution of grafts as shown in Fig. 6. In other words, branches can be distributed homogeneously or heterogeneously based on the reactivity ratio of the terminal functional group on the macromonomer and the low molecular weight monomer $[18,19]$.

Zhou et al. (2016) studied the role of alkyl grafting in enhancing the separation of VOCs from water in which ethyl acetate (EA) in a water mixture was selected for the study. Preparation of the alkyl grafted PDMS membranes was completed by different alkyl chain length through two single steps. First, a known quantity of alkyl groups was introduced into a linear polyhydromethylsiloxane (PHMS). Second, alkyl modified PHMS was cross-linked and grafted homogenously into vinyl terminated polydimethylsiloxane. The results confirmed that alkyl grafted PDMS separation performance depends on both the alkyl grafting ratio and alkyl chain length [20].

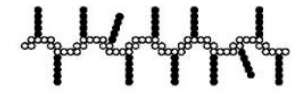

Homogeneous distribution of grafts

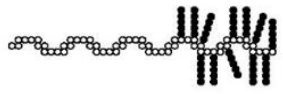

Heterogeneous distribution of grafts

Fig. 6. Different techniques of membrane alkyl grafting.

Investigations on the effect of alkyl grafting on both separation factor and total flux have been discussed in previous studies. The separation factor or selectivity increased initially and then decreased slightly with the increase in alkyl grafting ratio. Yet, an increase in the alkyl grafting ratio would decrease total and EA fluxes. Alkyl chain length also played a key role in the separation efficiency of EA from water. Higher alkyl chain length decreased both total flux and EA flux across the PDMS membrane due to the reduced free volume. Thus, C8-PDMS showed higher flux rates than those of C12-PDMS and C16-PDMS. Flux rates were associated with the free volume that is explained by the solubility and diffusivity of EA and water in membranes based on the solution-diffusion model. The octyl grafted PDMS membrane (C8-PDMS) showed an improved separation performance in removal of BA, MTBE, and $\mathrm{BuOH}$ from water [20]. Fig. 7 demonstrates the separation factor effectiveness of C8-PDMS and expected total fluxes for the treatment of the three different VOCs components from water.
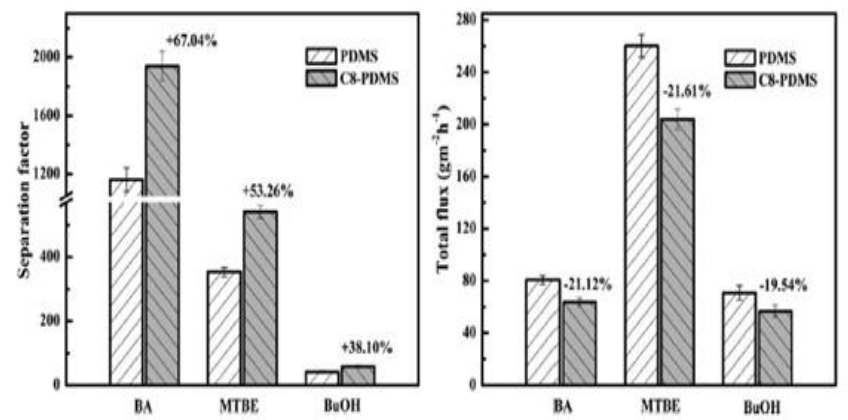

Fig. 7. Effect of alkyl grafting of C8- PDMS on separation factor and total flux for the treatment of different VOCs from water [20].

\section{B. Distillation membranes}

Electro-spun nanofiber membrane: Menkhaus et al. (2010) and Feng et al. (2012) initiated the practicality of electro-spun nanofiber membrane for the removal of VOCs compounds from water. Electrospinning is a much more advanced technique than conventional fiber spinning like melt-spinning, wet-spinning, and dry-spinning, which can only produce microfibers. Electrospinning technique creates nanofibers with enormous surface area in a small volume which allows for more mass transfer within the membrane [21,22].

Gas stripping electro-spun nanofiber membrane distillation system has been utilized for the removal of Chloroform from water, Fig. 8. The major components of the system were feed tank, membrane distillation cell, condenser (cold trap) and pumping system. $\mathrm{N}_{2}$ sweep gas was used to transport the separated Chloroform vapors into the cold trap for condensation. The membrane distillation cell characterized with a diameter of $13 \mathrm{~cm}$ and was made of high-pressure polyethylene. The membrane distillation cell was designed with two chambers ( $2 \mathrm{~cm}$ height per chamber), and they were the feed chamber and the stripping gas chamber in which the membrane was placed between both of them. The membrane effective area of about $19.6 \mathrm{~cm}^{2}$ was the contact area between the feed liquid (water) and the stripping gas $\left(\mathrm{N}_{2}\right)$. Chloroform/water mixture (feed) and $\mathrm{N}_{2}$ sweep gas were fed simultaneously and parallel into the MD cell. Chloroform vapors were removed from the water stream by the sweep gas and after being separated by the membrane. It has been shown that the system was able to decrease Chloroform from 1157 to 475 ppm in 5 hours with $\mathrm{N}_{2}$ sweep gas at $60^{\circ} \mathrm{C}$ [22].

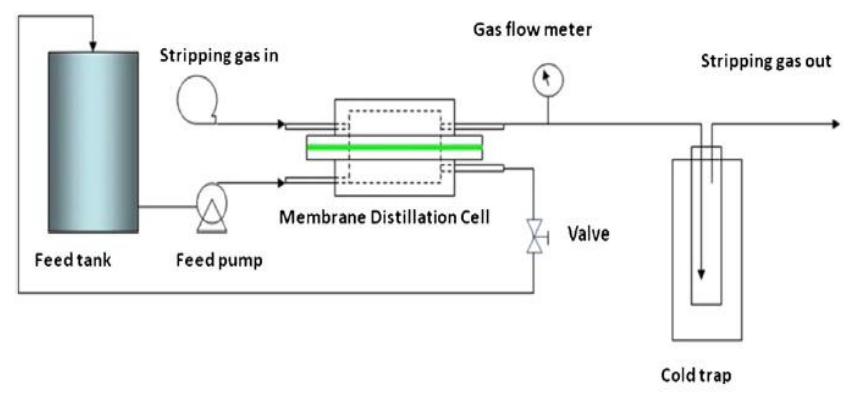

Fig. 8. Gas stripping electro-spun nanofiber MD system [22]. Vacuum Membrane Distillation (VMD): VMD is effective in the treatment of VOCs or volatile compounds from water by applying vacuum at the permeate side [16]. Transport mechanisms include three steps: evaporation at the liquid feed/membrane interface, diffusion of the vapor feed mixture through the membrane pores and condensation of the vapor stream on the downstream side [14].

Couffin et al. (1998) approved that VMD is a promising way for the removal of Halogenated VOCs (HOVs) such as Chloroform, trichloroethylene (TCE) and tetrachloroethylene. Factors that must be taken into consideration to increase the separation efficiency include, but not limited to, permeate flux, membrane selectivity, feed concentration, feed temperature, and system pressure. The estimated typical VMD flux was between 50 and $150 \mathrm{~L} / \mathrm{m}^{2} \mathrm{hr}$ [14].

The effect of the downstream pressure on the separation of TCE from a TCE/water mixture at different temperatures has been investigated and it was determined that both flux and 
selectivity were dependent on feed temperature and downstream pressure. It was found that total flux and selectivity had different responses to the latter mentioned variables. For example, total flux decreased and selectivity increased with increasing downstream pressure [14]. However, increasing the feed temperature increased total flux and decreased selectivity. Couffin experiment work showed high equivalent fluxes which suggested that VMD was and would be a promising way to remove TCE from water at low concentrations $[14,16]$.

\section{CONCLUSION}

Advancements in membrane technologies like pervaporation and membrane distillation directed scientific scholars to consider membranes for the treatment of VOCs from water and wastewaters. Currently, air stripping is the most common technique utilized for the treatment of VOCs from water since it can deal with a large variety of VOCs. However, air stripping is susceptible to fouling and is not effective for low volatile compounds. Further, most of the conventional treatment processes have a certain degree of shortcomings and limitations for the removal of VOCs.

Membrane pervaporation separation occurs due to the difference in the partial pressures of the components on the two sides where the membrane distillation process corresponds to both partial pressure and thermal gradients. Current advanced PV membranes include polysiloxaneimide (PSI) and polydimethylsiloxane (PDMS) where electro-spun nanofiber membrane and vacuum membrane distillation (VMD) are advanced MD membranes. Both PV and MD membranes showed great potential for the treatment of VOCs from water. Nevertheless, it is important to consider certain factors such as feed temperature, downstream pressure, component volatility (Henry's constant) and VOCs concentration to achieve the ideal treatment.

\section{ACKNOWLEDGMENT}

The author would like to thank Dr. Childress (from the University of Southern California) for her invaluable feedback and discussions during the MS coursework which greatly refined the research work.

\section{REFERENCES}

[1] Maddah, H.A. (2016). Modeling the Relation between Carbon Dioxide Emissions and Sea Level Rise for the Determination of Future (2100) Sea Level. American Journal of Environmental Engineering, 6(2), $52-61$.

[2] Maddah, H.; and Chogle, A. (2016). Biofouling in reverse osmosis: phenomena, monitoring, controlling and remediation. Applied Water Science, 1-15.

[3] Maddah, H.A. (2016). Optimal Operating Conditions in Designing Photocatalytic Reactor for Removal of Phenol from Wastewater. ARPN Journal of Engineering and Applied Sciences, 11(3), 1799-1802.

[4] Maddah, H.A.; and Chogle, A.M. (2015). Applicability of low pressure membranes for wastewater treatment with cost study analyses. Membrane Water Treat, 6(6), 477-488.

[5] Maddah, H.A. (2016). Application of Finite Fourier Transform and Similarity Approach in a Binary System of the Diffusion of Water in a Polymer. Journal of Materials Science and Chemical Engineering, 4(04), 20.

[6] Kujawa, J.; Cerneaux, S.; and Kujawski, W. (2015). Removal of hazardous volatile organic compounds from water by vacuum pervaporation with hydrophobic ceramic membranes. Journal of Membrane Science, 474, 11-19.

[7] Backer, W.S. (2013). The impact of methyl tertiary-butyl ether (MTBE) on contaminated drinking water in organic blood chemistry Impact of MTBE on organic blood chemistry. Journal of Saudi Chemical Society.

[8] Levchuk, I.; Bhatnagar, A.; and Sillanpää, M. (2014). Overview of technologies for removal of methyl tert-butyl ether (MTBE) from water. Science of The Total Environment, 476, 415-433.

[9] Eustache, H.; and Histi, G. (1981). Separation of aqueous organic mixtures by pervaporation and analysis by mass spectrometry or a coupled gas chromatograph-mass spectrometer. Journal of Membrane Science, $8(2), 105-114$.

[10] Rautenbach, R.; and Albrecht, R. (1985). The separation potential of pervaporation: Part 1. Discussion of transport equations and comparison with reverse osmosis. Journal of membrane science, 25(1), 1-23.

[11] Peng, M.; Vane, L.M.; and Liu, S. X. (2003). Recent advances in VOCs removal from water by pervaporation. Journal of hazardous materials, $98(1), 69-90$.

[12] PT. Gapura Liqua Mandiri Water Company (2010). Introduction to Pervaporation. Retrieved from http://www.liquamandiri.com/technology/pervaporation

[13] Zenon Technologies Inc. (1996). Zenon Cross-Flow Pervaporation Technology: Innovative Technology Evaluation Report. US Environmental Protection Agency, National Risk Management Research Laboratory Office of Research and Development.

[14] Couffin, N.; Cabassud, C.; and Lahoussine-Turcaud, V. (1998). A new process to remove halogenated VOCs for drinking water production: vacuum membrane distillation. Desalination, 117(1), 233-245.

[15] BLUE-tec Company (2016). Membrane Distillation: Principle of MD. Retrieved

from http://www.blue-technologies.nl/technologies-membranedistillation

[16] Karanikola, V.; Corral, A.F.; Mette, P.; Jiang, H.; Arnoldand, R.G.; and Ela, W.P. (2014). Solar membrane distillation: desalination for the Navajo Nation. Reviews on environmental health, 29(1-2), 67-70.

[17] Chang, Y.H.; Kim, J.H.; Lee, S.B.; and Rhee, H.W. (2000). Polysiloxaneimide membranes for removal of VOCs from water by pervaporation. Journal of applied polymer science, 77(12), 2691-2702.

[18] Shinoda, H.; and Matyjaszewski, K. (2001). Structural control of poly (methyl methacrylate)-g-poly (lactic acid) graft copolymers by atom transfer radical polymerization (ATRP). Macromolecules, 34(18), 6243-6248.

[19] Hawker, C.J.; Mecerreyes, D.; Elce, E.; Dao, J.; Hedrick, J.L.; Barakat, I.; and Volksen, W. (1997). "Living" free radical polymerization of macromonomers: Preparation of well defined graft copolymers. Macromolecular Chemistry and Physics, 198(1), $155-166$.

[20] Zhou, H.; Su, Y.; Chen, X.; Luo, J.; and Wan, Y. (2016). High-performance PDMS membranes for pervaporative removal of VOCs from water: The role of alkyl grafting. Journal of Applied Polymer Science, 133(31).

[21] Menkhaus, T.J.; Zhang, L.; and Fong, H. (2010). Applications of electrospun nanofiber membranes for bioseparations. Nova Science Publishers, Inc.

[22] Feng, C., Khulbe, K.C., and Tabe, S. (2012). Volatile organic compound removal by membrane gas stripping using electro-spun nanofiber membrane. Desalination, 287, 98-102.

Hisham A. Maddah is a lecturer in the Chemical Engineering Department at King Abdulaziz University (KAU) in Rabigh, KSA; he is currently sponsored by KAU to complete the PhD studies at the University of Illinois at Chicago (UIC). Mr. Maddah has received his BS and MS degrees in chemical engineering from KAU in 2012 and the University of Southern California (USC) in 2017, respectively. He has worked in the oil/gas industry for two years with the Chemical Business Department in Saudi Aramco (2012-2014) before shifting to academia in 2014. Mr. Maddah has published more than 30 articles/conference papers related to chemical engineering and higher education in reputed international peer-reviewed journals. His research interests are (1) Desalination; (2) Photovoltaics; (3) Nanotechnology; and (4) Higher education administration. 


\begin{tabular}{|c|c|}
\hline \multicolumn{2}{|c|}{ Nomenclatures } \\
\hline \multicolumn{2}{|r|}{ The effective area of the membrane, $m^{2}$} \\
\hline I & Total membrane permeation flux, $\mathrm{kg} / \mathrm{m}^{2} \mathrm{~h}$ \\
\hline$Q$ & Total amount of permeate collected by the membrane, $\mathrm{kg}$ \\
\hline$T$ & $\begin{array}{l}\text { Permeation time or the experimental time after the steady state } \\
\text { situation, hrr }\end{array}$ \\
\hline$W_{t y y}$ & Weight of a dry membrane, $g$ \\
\hline$W_{\text {wat }}$ & $\begin{array}{l}\text { Weight of a swollen membrane or membrane saturated with } \\
\text { organics, } g\end{array}$ \\
\hline$X_{w}$ & Weight fraction of organics in the feed (liquid) \\
\hline$X_{\mathrm{w}}$ & Weight fraction of water in the feed (liquid) \\
\hline$Y_{g}$ & Weight fraction of organics in the permeate (gas) \\
\hline$Y_{\mathrm{w}}$ & Weight fraction of water in the permeate (gas) \\
\hline \multicolumn{2}{|c|}{ Greek Symbols } \\
\hline$\alpha_{\text {id }}$ & Membrane selectivity of species $\tilde{i}$ relative to species $\tilde{i}$ \\
\hline$\Phi_{p}$ & Degree of membrane sorption, wt $\%$ \\
\hline \multicolumn{2}{|c|}{ Abbreviations } \\
\hline AGMD & Air Gap Membrane Distillation \\
\hline BTEX & Benzene, Toluene, Ethyl Benzene, and Xylenes \\
\hline TCE & Trichloroethylene \\
\hline DCMD & Direct Contact Membrane Distillation \\
\hline MD & Membrane Distillation \\
\hline PCE & Tetrachloroethylene \\
\hline PDMS & Polydimethylsiloxane \\
\hline PSI & Polysiloxaneimide \\
\hline PV & Pervaporation \\
\hline SGMD & Sweep Gas Membrane Distillation \\
\hline UF & Ultra-Filtration \\
\hline VMD & Vacuum Membrane Distillation \\
\hline VOCs & Volatile Organic Compounds \\
\hline RO & \\
\hline
\end{tabular}

